\title{
Gingival Crevicular Blood Glucose Levels. Is it a Reliable Tool for Screening Diabetes in a Dental Office?
}

\author{
Abhijeet R Sande ${ }^{1}$, Sanjeela Guru², Rakshith Guru ${ }^{3}$, Satish Gaduputi ${ }^{4}$, Divya K Thati ${ }^{5}$, Srinivasa T Siddeshappa ${ }^{6}$
}

\begin{abstract}
Aim: Periodontal disease is associated with gingival bleeding. Thus, the present study was aimed towards evaluating, whether the blood oozing during routine periodontal examination can be used for evaluating blood glucose levels.

Materials and methods: A total of 100 patients reporting to Department of Oral Medicine and Radiology, within the age group of 25-55 years were enrolled. In fasting condition, gingival crevicular blood (GCB), finger-stick blood (FSB) was measured using a glucometer and venous blood (VB) glucose values were measured in the laboratory with glucose oxidase method. All the parameters thus recorded were then subjected to statistical analysis.

Results: When compared, the GCB glucose values, VB glucose values and the FSB glucose values measured with glucometer in the diabetic and nondiabetic group were found to be highly statistically significant $(p=0.000)$.

Conclusion: Gingival crevicular blood collected during diagnostic periodontal examination can provide a valid and acceptable source for measuring blood glucose levels using a glucometer. In addition, the technique described is quick, safe, easy to perform and more acceptable to the patients.

Clinical significance: Dental surgeon can play an active role in screening of patients for glucose levels during routine periodontal examination and help to increase the frequency of diabetes screening in dental offices especially in undiagnosed diabetic cases which can be referred to the physician for appropriate management of the condition.

Keywords: Gingival crevicular blood, Glucometer, Glucose oxidase.

The Journal of Contemporary Dental Practice (2020): 10.5005/jp-journals-10024-2752
\end{abstract}

\section{INTRODUCTION}

Indicators of health must be regularly monitored to remain healthy and prevent problems from becoming worse. This can be done by regular blood checkups. However patients frequently are unaware that a dentist may need a medical consultation, a prerequisite to begin dental treatment and thus are reluctant to solicit medical evaluation. ${ }^{1}$ Due to this practical yet erroneous psychology of the population, routine health checkup is extremely rare. Hence it would be helpful if we employ a technique which is safe, easy, painless and comfortable for the patient for screening of systemic diseases in the dental setup.

Diabetes mellitus is a chronic endocrine disorder and represents a major health burden faced by the society today. There is a steep rise in incidence of diabetes worldwide and even more particularly, in India. ${ }^{2}$ Moreover, $50 \%$ of diabetics go undiagnosed. ${ }^{2}$ Diabetes mellitus is associated with several complications. Recent evidence has indicated that these complications may begin several years before the diagnosis of diabetes mellitus is established. Thus the key to minimizing long-term complications of diabetes resulting in morbidity and mortality of these patients is early diagnosis. ${ }^{3}$

It is the responsibility of the dental practitioner to screen for physical conditions which may alter the dental treatment or the general well-being of their patients. Thus, in addition to maintaining the oral health of the people with diabetes, dentists may also play a role in screening for disease in the general population. One technique for screening diabetes mellitus in the dental setup itself would be by using gingival crevicular blood (GCB). ${ }^{4}$ Gingival crevicular blood elicited during routine periodontal probing of inflamed tissue has been used previously to test the blood glucose levels. ${ }^{4-6}$ The blood glucose levels obtained from GCB have shown
${ }^{1}$ Department of Oral Medicine and Radiology, School of Dental Sciences, Krishna Institute of Medical Sciences Deemed University, Karad, Maharashtra, India

${ }^{2}$ Department of Periodontics, Vydehi Institute of Dental Sciences and Research Centre, Bengaluru, Karnataka, India

${ }^{3}$ Department of Prosthodontics, ESIC Dental College, Gulbarga, Karnataka, India

${ }^{4,5}$ Department of Conservative Dental Sciences, Ibn Sina National College for Medical Studies, Jeddah, Kingdom of Saudi Arabia

${ }^{6}$ Department of Periodontology, ESIC Dental College, Gulbarga, Karnataka, India

Corresponding Author: Sanjeela Guru, Department of Periodontics, Vydehi Institute of Dental Sciences and Research Centre, Bengaluru, Karnataka, India, e-mail: sanjeelaguru@yahoo.co.in

How to cite this article: Sande AR, Guru S, Guru R, et al. Gingival Crevicular Blood Glucose Levels. Is it a Reliable Tool for Screening Diabetes in a Dental Office? J Contemp Dent Pract 2020;21(4):421-425.

Source of support: Nil

Conflict of interest: None

to correlate to the blood glucose levels obtained from peripheral finger puncture. ${ }^{4-7}$ However literature which compares GCB to venous blood (VB) is sparse. Thus, the present study attempts to check how accurately the GCB reflects blood glucose levels of capillary and VB.

Since periodontal disease itself is associated with gingival bleeding and the dental practitioner is more familiar with this extravasated blood which is produced during routine diagnostic procedures, this extravasated blood is far more atraumatic than 
using a lancet for finger puncture. ${ }^{5}$ This blood extravasating during routine periodontal examination can be a potential source for the estimation of blood glucose. Thus, this study was undertaken to assess whether the blood oozing during routine periodontal examination can be used for accurately evaluating blood glucose levels by comparing it with capillary and VB.

\section{Materials and Methods}

A total of 130 patients reporting to Department of Oral Medicine and Radiology, School of Dental Sciences, KIMSDU, Karad were screened of which 100 patients within the age group of 25-55 years and who fulfilled the inclusion criteria were recruited into the study. The study was approved by the institutional review board. A written informed consent was obtained from all the participants prior to the study.

Patients with moderate to severe gingivitis in the maxillary anterior region and who extravasated enough blood in the same region during routine periodontal probing were included in the study. Patients already diagnosed as diabetic or on medication for the same were excluded from the study. Patients who were systemically compromised (severe cardiovascular, hepatic, immunologic, renal, hematologic, or other organ disorders), patients with a history of taking vitamin $C$ supplement, and pregnant females were also excluded from the study. Patients unaware of their diabetic status were included in the study.

\section{Gingival Crevicular Blood Sampling}

Since the gingiva in relation to maxillary anterior teeth offers more ideal access for sample collection, we chose gingiva related to maxillary anterior teeth, i.e., 13-23 for blood sample collection. Isolation was done with cotton rolls and salivary contamination was prevented by using gauze, absorbent papers and air-drying. The selected site was probed with William's graduated periodontal probe and the blood extravasating from the gingival crevice was placed against the top edge of the test strip. The blood by capillary action was drawn automatically into the strip reaction cell, until the confirmation window displayed full. Caution was taken to obtain the blood sample on the reagent strip without contacting gingiva or the teeth (Fig. 1A).

The blood glucose value which was displayed by the glucometer (One Touch Select Plus Lifescan Scotland Ltd.) was recorded immediately as GCB glucose value.

\section{Finger-stick Blood Sampling}

Following the crevicular blood sampling, another blood sample was obtained from the pulp of fourth finger on the left hand. The finger was disinfected by wiping with surgical spirit followed by finger puncture using a sterile lancet. First drop of blood was wiped away and the second drop of blood obtained was held against the test strip of the glucometer (Fig. 1B) and the values displayed were recorded as Finger-stick blood (FSB) glucose value.

\section{Venous Blood Sampling}

About $3 \mathrm{~mL}$ of VB sample for each patient was collected from the antecubital vein. A drop of VB was held against the test strip of the glucometer and the reading was noted as VB glucose level measured with glucometer (Fig. 1C).

The rest of the VB approximately $(3 \mathrm{~mL})$ was centrifuged to obtain plasma which was analyzed for glucose levels by laboratory method. The values obtained from this method were recorded as VB glucose level measured with glucose oxidase method.

All the glucose values for gingival, capillary and VB were recorded by single researcher. The blood collection was done in the morning, i.e., between 8 am and 9 am in fasting condition.

Based on the values recorded the subjects were divided into two groups:

- Nondiabetics-fasting blood glucose values $\leq 126 \mathrm{mg} / \mathrm{dL}$.

- Diabetics-fasting blood glucose values $>126 \mathrm{mg} / \mathrm{dL}$.

\section{Statistical Analysis}

All the parameters recorded were tabulated and subjected to statistical analysis using Statistical procedure for social services (SPSS version 16 for windows).

\section{Results}

The distribution of study samples by gender and age is shown in Table 1. The study shows that out of the 69 males and 31 females, 49 males and 21 females were diabetic and 20 males and 10 females were found to be nondiabetic. The mean age and standard deviation of the male and female subjects with respect to the entire sample size $(n=100)$ is also shown in Table 1 , where the mean age of the subjects in the diabetic group was 40.96 and the nondiabetic group was 39.80 .

Comparison of diabetic and nondiabetic patients measured with VB on glucometer and glucose oxidase method by Student $t$ test shows the data to be statistically significant $(p=0.000)$ as shown in Table 2. The significance of this test validates the use of glucometer to get correct values as compared with the laboratory values.

Correlation between blood glucose measured with gingival blood, peripheral finger-stick and VB on glucometer and VB measured with glucose oxidase method by Karl Pearson's correlation method shows it to be highly statistically significant ( $p=0.000$ ) (Table 3). This test validates the use of gingival blood for glucose estimation which is more significant than use of FSB.
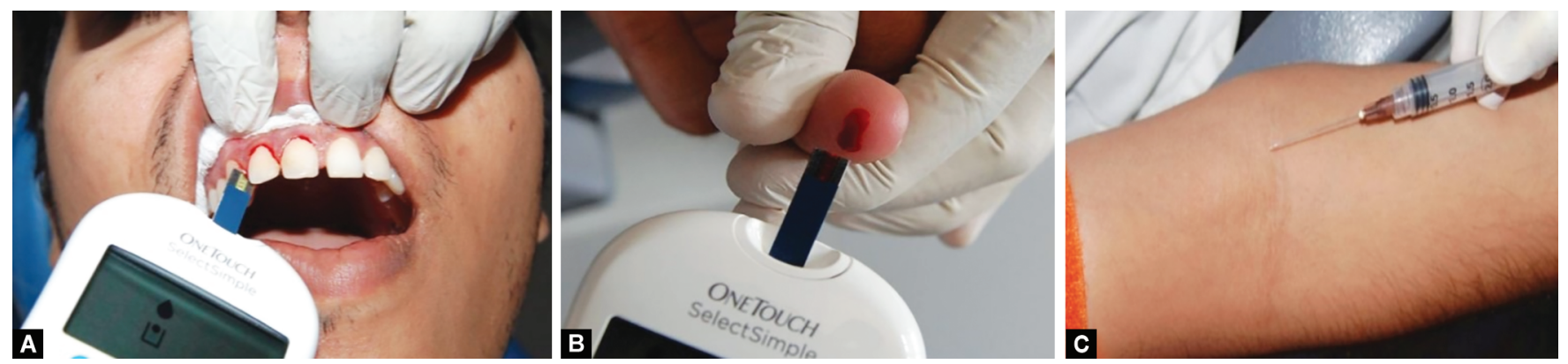

Figs 1 A to C: Sampling gingival crevicular blood (A), fingerstick blood (B) and venous blood (C) 
Table 1: Gender and age distribution of study samples

\begin{tabular}{|c|c|c|c|c|c|c|c|}
\hline \multirow[b]{2}{*}{ Group } & \multicolumn{5}{|c|}{ Gender } & \multicolumn{2}{|c|}{ Age } \\
\hline & Male & $\%$ & Female & $\%$ & Total & Mean age & $S D$ \\
\hline Diabetics & 49 & 70.00 & 21 & 30.00 & 70 & 40.96 & 7.35 \\
\hline Non-diabetics & 20 & 66.67 & 10 & 33.33 & 30 & 39.80 & 8.96 \\
\hline Total & 69 & 69.00 & 31 & 31.00 & 100 & 40.61 & 7.84 \\
\hline
\end{tabular}

Table 2: Comparison of diabetics and non-diabetics measured with venous blood on glucometer and glucose oxidase method by student $t$ test

\begin{tabular}{lllllll}
\hline Variables & Group & $n$ & Mean & SD & tvalue & $p$ value \\
\hline Venous blood glucose value measured with glucometer & Diabetics & 70 & 145.3143 & 8.7120 & 20.9417 & $0.0000^{*}$ \\
& Non-diabetics & 30 & 106.0000 & 8.3377 & \\
Venous blood glucose value measured with glucose oxidase & Diabetics & 70 & 146.4000 & 8.4380 & 21.8237 & $0.0000^{*}$ \\
method & Non-diabetics & 30 & 106.4333 & 8.2824 & & \\
Difference & Diabetics & 70 & -1.0857 & 1.2128 & -2.3482 & $0.0209^{* *}$ \\
& Non-diabetics & 30 & -0.4333 & 1.4065 & & \\
\hline
\end{tabular}

*Statistically significant

${ }^{*} p<0.01,{ }^{* *} p<0.05$

Table 3: Correlation between blood glucose measured with gingival blood, peripheral fingerstick and venous blood on glucometer and venous blood measured with glucose oxidase method by Karl Pearson's correlation method

\begin{tabular}{llll}
\hline Correlation between & rvalue & t value & $p$ value \\
\hline $\begin{array}{l}\text { Gingival blood glucose value with } \\
\text { venous blood glucose value on } \\
\text { glucometer }\end{array}$ & 0.9975 & 139.0523 & $0.0000^{*}$ \\
$\begin{array}{l}\text { Gingival blood glucose value } \\
\text { with venous blood glucose value } \\
\text { measured with glucose oxidase } \\
\text { method }\end{array}$ & 0.9980 & 156.1111 & $0.0000^{*}$ \\
$\begin{array}{l}\text { Finger stick blood glucose value with } \\
\text { venous blood glucose values on } \\
\text { glucometer }\end{array}$ & & & \\
$\begin{array}{l}\text { Finger stick blood glucose value } \\
\text { with venous blood glucose value } \\
\text { measured with glucose oxidase } \\
\text { method }\end{array}$ & 0.9969 & 114.2390 & $0.0000^{*}$ \\
$\begin{array}{l}\text { Gingival blood glucose value and } \\
\text { finger stick blood glucose value }\end{array}$ & 0.9923 & 2.1167 & $0.03^{* *}$ \\
\hline
\end{tabular}

*Statistically significant

$r$, denotes correlation coefficient

${ }^{*} p<0.01,{ }^{* *} p<0.05$

Linear regression analysis was calculated for the mean GCB glucose values against the VB glucose values on glucometer (Fig. 2). From the values plotted in Figure 2 we can propose the following formula:

Venous blood on glucometer $=1.0071$ (blood glucose measured with gingival blood) -0.064 .

During screening of the patients, 70 patients were found to have glucose levels higher than the normal fasting range and were referred to the physician for consultation.

Thus, from the results of the present study we can infer that gingival blood for glucose estimation was more significant than use of fingerstick blood and it was comparable to VB glucose values.

\section{Discussion}

Diabetes Mellitus is the most common systemic disease encountered by a dentist and over a century, various methods have

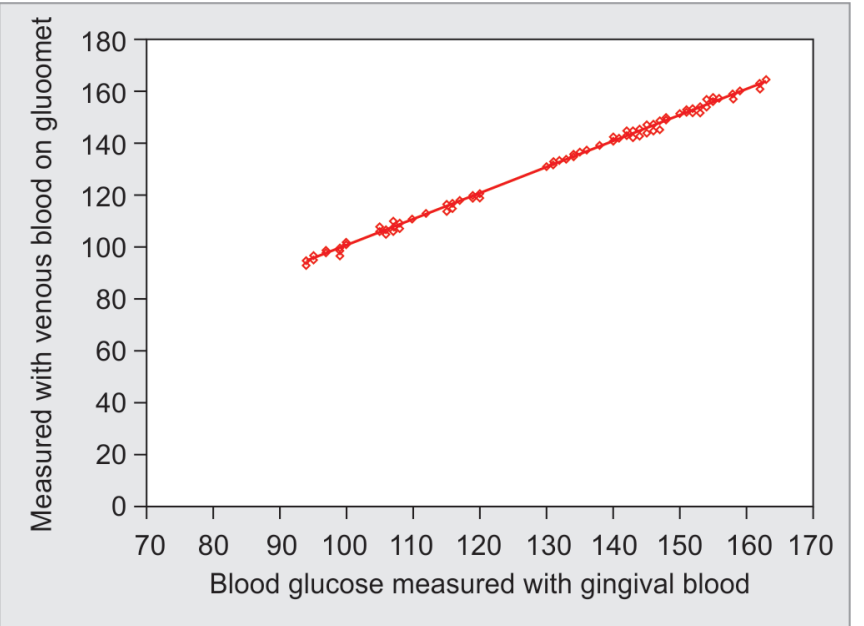

Fig. 2: Linear regression analysis

been used to measure glucose level in biological fluids, but the search for more specific, sensitive and simple method continues. Clinicians routinely send VB, or urine samples for determining glucose levels to clinical biochemistry laboratories since decades. But of late portable glucose monitors (glucometers) are used both as a bedside testing of glucose in a hospital as well as for home testing done by patients themselves. ${ }^{4,8}$ Since the glucometer is rampantly available the present study was done to check if it is possible to use the same glucometer to monitor the blood glucose levels in patients who extravasate small amount of blood during routine periodontal examination.

It is widely accepted that patients with diabetes mellitus exhibit more severe periodontal disease and gingival bleeding is more severe, which has been conformed in extensive reviews. ${ }^{9-11}$ This is more pronounced in poorly controlled diabetics and patient's with undiagnosed diabetes.

Finger puncture using a lancet is painful and hence quite traumatic. Extravasation of blood during periodontal examination is painless, atraumatic and unnoticed by the patient. Thus, procedure of collection of blood for glucose estimation becomes a simple affair. Hence in the present study we used glucometer for detecting blood glucose levels from blood extravasated from the 
gingival crevice and compared it with capillary and VB glucose levels measured with glucose oxidase (laboratory) method which is considered as gold standard.

In a study conducted by Chopra et al. ${ }^{12}$ lower anterior teeth were used to collect the blood sample which is in contrast with our study where upper anterior teeth are used to prevent contamination and pooling of saliva.

Our study involves free flowing blood from periodontal probing which is atraumatic and in contrast to puncturing the interdental papillae with a sharp lancet to obtain blood which would be traumatic to the patient. In the study conducted by Parker et al. ${ }^{13}$ they used plastic pipette for collection of blood from gingival crevice which is in contrast with present study where blood was directly used for glucose estimation to prevent the complication of contamination and also procedure can easily be replicated in the dental setup. In contrast to the above study, the sampling procedure used in the present study had advantages of it being easier to perform, was less time-consuming and did not require any additional equipment to collect GCB.

One study suggested that a blood laden subgingival curette be used to rub blood onto the glucometer test strip. ${ }^{14}$ However this method of rubbing or rather direct wiping of intraoral blood on the surface of the glucometer test strip may not only damage the chemical indicator surface of the strip but also hinder production of a uniformly timed reaction. Tsutsui et al. also suggested that this method can cause significant contamination of the sample by saliva or oral debris present at gingival site or from the curette which could collect plaque and gingival crevicular fluid when it enters into the gingival sulcus. ${ }^{14}$ In our study, free flowing blood was collected directly on to the strip, which was placed passively against the hemorrhaging area. Hence, chances of contamination were minimized.

Most of the recently developed glucometer require very small amount of blood ( $2-3 \mu \mathrm{L})$. Glucose measurement using a self-monitoring device (glucometer) is achievable even if there is very low gingival crevicular bleeding, as the current glucometers allows glucose level estimation even in small amount of blood, i.e., as low as $1 \mu \mathrm{L} .{ }^{4,15}$

In the present study in order to allow comparison of glucometer values with that of the laboratory derived values, the glucometer was plasma calibrated thus negating the need for calibration to whole blood glucose. During screening of the patients, 70 patients were found to have glucose levels higher than the normal fasting range and were referred to the physician for consultation.

In order to get the predictability of VB glucose value at a given GCB glucose value in an individual patient, linear regression analysis was done. A formula was derived based on linear regression analysis which is:

Venous blood glucose value $=1.0071$ (gingival blood glucose level) -0.064

The accuracy of the glucometer derived GCB glucose readings from each patient was verified by correlating the glucose readings derived through a traditional finger-stick sample and VB glucose value from the patient. In the present study there was a strong statistically significant correlation between gingival blood glucose values (measured on glucometer) and VB glucose values measured with glucose oxidase (laboratory) method ( $r=0.998$, $p<0.00)$ in both diabetics and nondiabetics. The correlation between FSB glucose values and VB glucose values measured with glucose oxidase method was $r=0.996, p<0.001$ for diabetics and nondiabetics. The results of the present study are similar to the findings of the studies conducted previously by Parihar et al., Shetty et al. and Bhavsar et al., ${ }^{76,17}$ These correlations were more significant than those obtained by Parker et al. ${ }^{13}$ The higher correlation obtained in our study might be due to the second generation glucose monitors used in this study compared with first generation monitors used in earlier studies. In the present study a self timing second generation glucometer approved by the Federation Dentaire International (FDI) was used for better accuracy. This glucometer has the advantage that it requires very small quantity of blood $(1 \mu \mathrm{L})$ for glucose detection thus is helpful even in cases with very mild gingival bleeding. Since the meter is plasma calibrated, the necessity for calibration to whole blood glucose is eliminated and hence allows comparison of glucometer values directly with laboratory derived values.

From the results and within the limitations of the present study, we can suggest that GCB which extravasates from routine periodontal examination can be an excellent source of blood for analysis of blood glucose levels. Patients can reliably be screened for diabetes by measuring glucose levels in GCB. This method is attractive to the dental practitioner because of its simplicity of use and the fact that the method is relatively inexpensive yet provides sufficiently accurate results. Thus, it can be effectively used as an in-office screening method for patients who are suspected to have diabetes. However further studies employing a larger sample size may be conducted to validate GCB glucose as a reliable source for monitoring blood glucose levels.

\section{Conclusion}

Thus, it can be suggested that GCB collected during diagnostic periodontal examination can be used as an acceptable method for measuring blood glucose levels with the help of a glucometer. In addition, this technique is not only quick and easy to perform but also safe and more atruamatic to the patients.

A dental surgeon can play active role in screening of patients for glucose levels during routine periodontal examination and help to increase the frequency of diabetes screening in dental offices. This would add value especially in patient's undiagnosed diabetes as they can be referred to the physician for appropriate management of the condition.

\section{References}

1. Manski RJ, Hoffmann D, Rowthorn V. Increasing access to dental and medical care by allowing greater flexibility in scope of practice. Am J Public Health 2015;105(9):1755-1762. DOI: 10.2105/AJPH.2015.302654.

2. Wild S, Roglic G, Green A, et al. Global prevalence of diabetes: estimates for the year 2000 and projections for 2030. Diabetes Care 2004;27(5):1047-1053. DOI: 10.2337/diacare.27.5.1047.

3. Chentli F, Azzoug S, Mahgoun S. Diabetes mellitus in elderly. Indian J Endocrinol Metab 2015;19(6):744-752. DOI: 10.4103/22308210.167553.

4. Sibyl S, Bennadi D, Kshetrimayum N, et al. Correlations between gingival crevicular blood glucose and capillary blood glucose: a preliminary report. J Lab Physicians 2017;9(4):260-263. DOI: 10.4103/ JLP.JLP_141_16.

5. Debnath $P$, Govila V, Sharma $M$, et al. Glucometric assessment of gingival crevicular blood in diabetic and non-diabetic patients: a randomized clinical trial. J Oral Biol Craniofac Res 2015;5(1):2-6. DOI: 10.1016/j.jobcr.2014.12.004.

6. Rajesh KS, Irshana R, Arun Kumar MS, et al. Effectiveness of glucometer in screening diabetes mellitus using gingival crevicular 
blood. Contemp Clin Dent 2016;7(2):182-185. DOI: 10.4103/0976237X.183072.

7. Shetty N, Shankarapillai R, Mathur LK, et al. Gingival crevicular blood: as a non-invasive screening tool for diabetes mellitus in dental clinics. J Indian Soc Periodontol 2013;17(4):472-477. DOI: 10.4103/0972124X.118319.

8. Ginsberg BH. Factors affecting blood glucose monitoring: sources of errors in measurement. J Diabetes Sci Technol 2009;3(4):903-913. DOI: $10.1177 / 193229680900300438$

9. Preshaw PM, Bissett SM. Periodontitis: oral complication of diabetes. Endocrinol Metab Clin North Am 2013;42(4):849-867. DOI: 10.1016/ j.ecl.2013.05.012.

10. Chang PC, Lim LP. Interrelationships of periodontitis and diabetes: a review of the current literature. J Dent Sci 2012;7(3):272-282. DOI: 10.1016/j.jds.2012.02.002.

11. Preshaw PM, Alba AL, Herrera D, et al. Periodontitis and diabetes: a two-way relationship. Diabetologia 2012;55(1):21-31. DOI: 10.1007/ s00125-011-2342-y.
12. Chopra P, Kumar T. Correlation of glucose level among venous, gingival and finger-prick blood samples in diabetic patients. Indian Soc Periodontol 2011;15(3):288-291. DOI: 10.4103/0972-124X.85678.

13. Parker RC, Rapley JW, Isley W, et al. Gingival crevicular blood for assessment of blood glucose in diabetic patient. J Periodontol 1993;64(7):666-672. DOI: 10.1902/jop.1993.64.7.666.

14. Tsutsui P, Rich SK, Schonfeld SE. Reliability of intraoral blood for diabetes screening. J Oral Med 1985;40(2):62-66.

15. Yared Z, Aljaberi K, Renouf N, et al. The effect of blood sample Volume on 11 glucose monitoring systems. Diabetes Care 2005;28(7): 1836-1837. DOI: 10.2337/diacare.28.7.1836.

16. Parihar S, Tripathi R, Parihar AV, et al. Estimation of gingival crevicular blood glucose level for the screening of diabetes mellitus: a simple yet reliable method. J Oral Biol Craniofac Res 2016;6(3):198-203. DOI: 10.1016/j.jobcr.2016.05.004.

17. Bhavsar MV, Brahmbhatt NA, Sahayata V, et al. Gingival crevicular blood for screening of blood glucose level in patients with and without diabetes: a chair-side test. Int J Dent Hyg 2016;14(2):92-97. DOI: 10.1111/idh.12139. 